\title{
"Extracto orujo de uva Malbec como inhibidor natural de la corrosión de materiales metálicos"
}

\section{"Malbec grape pomace extract as a natural inhibitor of the corrosion of metallic materials"}

Presentación: 06-07/10/2020

\section{Doctorando:}

\section{Patricia Silvana Carrizo}

Departamento Electromecánica, Laboratorio Metalurgia, Área de Arqueometalurgia, Facultad Regional Mendoza, Universidad Tecnológica Nacional-Argentina.

patricia.carrizo@frm.utn.edu.ar

\section{Director/a:}

\section{Cecilia Deyá}

\section{Co-director/a:}

\section{Ariel Fontana}

\section{Resumen}

La corrosión es un proceso de deterioro o destrucción de metales que, aunque inevitable, es factible de ser controlado. Debido a la toxicidad de las sustancias tradicionalmente utilizadas como inhibidoras del proceso corrosivo, hay un creciente interés en explorar inhibidores eficientes, económicos y amigables con el medio ambiente. El objetivo de este trabajo es evaluar la eficiencia anticorrosiva del extracto de orujo de uva Malbec (Vitis vinífera) frente a la corrosión de acero SAE 1010 en medio cloruro de sodio. El extracto fue obtenido mediante 2 métodos de extracción y se seleccionó aquel de mayor contenido de fenoles totales con método de Folin-Ciccolteau y el de mayor capacidad antioxidante mediante (2,2-azino-bis-(3-etilbenzotiazolin-6-sulfonato de amonio) ABTS. Para evaluar la eficiencia anticorrosiva se realizaron la evaluación del potencial de corrosión y ensayos de polarización del sustrato en suspensión de extracto orujo de uva en $\mathrm{NaCl}$. Como blancos se utilizaron soluciones de cloruro de sodio $0,5 \mathrm{M}$, respectivamente. Los resultados obtenidos mostraron que la presencia de orujo puede inhibir la corrosión del acero SAE 1010 inmerso en CINa al disminuir la velocidad de corrosión.

Palabras clave: Corrosión, Inhibidores, Extractos, Renovables, Electroquímica.

\begin{abstract}
Corrosion is a process of deterioration or destruction of metals that, although inevitable, is feasible to be controlled. Due to the toxicity of substances traditionally used as inhibitors of the corrosive process, there is a growing interest in exploring efficient, economical and environmentally friendly inhibitors. The objective of this work is to evaluate the anticorrosive efficiency of Malbec grape pomace extract (Vitis vinifera) against the corrosion of SAE 1010 steel in a sodium chloride medium. The extract was obtained by 2 extraction methods and the one with the highest total phenol content was determined with the Folin-Ciccolteau method and the one with the highest antioxidant capacity determined using ABTS (2,2-azino-bis- (3-
\end{abstract}


ethyl-benzothiazolin-6 -ammonium sulfonate) To evaluate the anticorrosive efficiency, the evaluation of the corrosion potential and polarization tests of the substrate in suspension of grape pomace extract in $\mathrm{NaCl}$ were carried out. $0.5 \mathrm{M}$ sodium chloride solutions were used. The results obtained showed that the presence of pomace can inhibit the corrosion of SAE 1010 steel immersed in $\mathrm{NaCl}$ by reducing the corrosion rate.

Keywords: Corrosion, Inhibitors, Extracts, Renewables, Electrochemistry.

\section{Introducción}

Diversos métodos pueden ser empleados para controlar el proceso de deterioro de metales denominado corrosión. El empleo de cubiertas orgánicas y/o inhibidores solubles en el medio corrosivo son las alternativas comúnmente utilizadas debido a los bajos costos, fácil aplicación y mantenimiento (Romagnoli \& Vetere, 1995). Los pigmentos anticorrosivos a base de fosfatos están siendo cuestionados debido a que el anión causa eutroficación (proceso natural y/o antropogénico) en reservas de agua dulce (Gerhard \& Bittner, 1986) y, además, algunos de ellos poseen cationes pesados como cinc o estroncio cuyo uso también ha sido restringido (Bittner,1989). Entre las alternativas para reemplazar total o parcialmente estos pigmentos se encuentran los extractos vegetales de plantas porque son biodegradables y renovables (Shirmohammadlia, Efhamisisia \& Pizzi, 2018). La industria vitivinícola genera como residuo sólido el orujo de uva el cual está compuesto por semillas, hollejos y escobajos. Estos residuos tienen una riqueza cualitativa y cuantitativa en constituyentes fenólicos, también conocidos como antioxidantes naturales (Alonso, 2000). Los extractos vegetales son una mezcla compleja de diferentes compuestos, a menudo estrechamente relacionados. Entre ellos se puede mencionar un grupo ampliamente distribuido en el reino vegetal, los polifenoles (familia que incluye los taninos, flavonoides, terpenoides, entre otros), muchos de estos compuestos pueden actuar como inhibidores orgánicos de la corrosión (Kesavan, Gopiraman \& Sulochana, 2012). Mendoza es la principal región vitivinícola argentina. En ella se concentra el $86 \%$ de los cultivos de Malbec, con 34.095,40 has. Malbec Luján de Cuyo es la primera zona con Denominación de Origen Controlada de América. El Malbec de esta sub-región presenta un color muy intenso y oscuro de tipo rojo cereza que puede llegar a parecer casi negro. El extracto se puede obtener tanto del orujo de uvas tintas como blancas, aunque según los reportes de la literatura las uvas tintas tendrían mejores propiedades antioxidantes (Ciudad \& Valenzuela, 2002). Resulta fundamental conocer el poder antioxidante del extracto de orujo de uva para poder determinar su eficiencia como inhibidor de la corrosión de aplicación tipo imprimante sobre materiales metálicos. El objetivo de este trabajo es caracterizar y evaluar mediante técnicas electroquímicas a los extractos de orujo de uva (Vitis vinífera), como inhibidor de la corrosión del acero SAE 1010 en medio cloruro de sodio.

\section{Desarrollo}

Obtención de los extractos

El orujo se obtuvo durante la etapa de trasiego a partir de uva Malbec (Vitis vinífera). A este orujo integrado por hollejos, semillas y escobajo se lo dejó secar al sol y se molió. Luego se trabajó con dos técnicas de extracción diferentes. En un caso, se pusieron en contacto $5 \mathrm{~kg}$ de orujo molido y seco con $5 \mathrm{~L}$ de alcohol etílico al $98 \%$ para una técnica de escala semi-piloto, y en el otro,se usaron $40 \mathrm{~g}$ de orujo molido y seco con $250 \mathrm{~mL}$ de alcohol etílico $+250 \mathrm{~mL}$ de agua destilada. En ambos casos se homogenizó la mezcla, y se colocó en baño ultrasónico manteniendo la temperatura entre $42-57^{\circ} \mathrm{C}$ para no afectar los compuestos polifenólicos. Posteriormente, se centrifugó todo el contenido, se filtró a vacío y se usó el rotavapor con el objetivo de concentrar el extracto.

Caracterización de los Extractos de orujo de uva.

A los dos extractos obtenidos se los caracterizó mediante: Contenido de Fenoles Totales por método de Folin-Ciccolteau y Actividad o Poder Antioxidante por ABTS (2,2'-azino-bis- (3-etil benzotiazolin-6-sulfonato de amonio). 
Contenido de fenoles totales (CFT)

La determinación del contenido de fenoles totales se realizó por el método colorimétrico Folin-Ciocalteu, según lo informado por (Antoniolli, Fontana, Piccoli \& Bottini, 2015). El contenido de fenoles totales se midió espectrofotométricamente con un espectrofotómetro UV-vis Cary-50 (Varian Inc., Mulgrave, Australia).A la muestra de extracto se le adicionó $50 \mu \mathrm{L}$ de ácido gálico como estándar para obtener una dilución 1:20. Se agregó $5 \mathrm{~mL}$ de agua destilada y se le adicionó $1250 \mu \mathrm{L}$ del reactivo de Folin. Se dejaron pasar entre 1 a 8 minutos, luego se adicionó $3,75 \mu \mathrm{L}$ de $\mathrm{Na}_{2} \mathrm{CO}_{3}(20 \mathrm{~g} / \mathrm{mL})$ y se guardó en ausencia de luz entre 1h 30' a $2 \mathrm{~h}$, con el objetivo de obtener lecturas de absorbancia a 750 y a $765 \mathrm{~nm}$ con una cubeta de $1 \mathrm{~cm}$ de camino óptico frente a un blanco preparado con agua destilada. Los resultados se expresaron como miligramos equivalentes de ácido gálico/gramo de extracto (mg GAE/g de materia seca) a partir de curvas de calibración realizadas con las soluciones estándar (tres repeticiones) en el rango entre 20 y $200 \mathrm{mg} / \mathrm{L}\left(\mathrm{R}^{2}=0,998\right.$ y $\mathrm{R}^{2}=0,999$, respectivamente para cada longitud de onda).

\begin{tabular}{|l|c|c|}
\hline \multicolumn{1}{|c|}{ Extractos Orujo de Uva } & F-C (Absorbancia: 750 nm) & F-C (Absorbancia: 765 nm) \\
\hline A (100\% etanol) & $12,0 \mathrm{mg} \mathrm{GAE} \mathrm{/g}$ & $11,9 \mathrm{mg} \mathrm{GAE} \mathrm{/g}$ \\
\hline B (50\% etanol -50\% agua) & $19,6 \mathrm{mg} \mathrm{GAE} \mathrm{/} \mathrm{g}$ & $19,5 \mathrm{mg} \mathrm{GAE} \mathrm{/g}$ \\
\hline
\end{tabular}

Tabla 1. Contenido de Fenoles Totales en cada extracto.

Evaluación de la actividad antioxidante

Se fundamenta en la cuantificación de la decoloración del radical ABTS•+, debido a la interacción con especies donantes de hidrógeno o de electrones. El radical catiónico ABTS•+ es un cromóforo que absorbe a una longitud de onda de $734 \mathrm{~nm}$ y se genera por una reacción de oxidación del ABTS•+ (2,2-azino-bis-(3-etil-benzotiazolin-6-sulfonato de amonio) con persulfato de potasio. Para el ensayo ABTS, el método descrito por (Ferreyra, Antoniolli, Bottini \& Fontana, 2019) fue empleado.

El catión radical ABTS (ABTS•+) se produjo mezclando 2,5 mL de solución madre de ABTS 7 mM y $44 \mu \mathrm{L}$ de $\mathrm{K}_{2} \mathrm{~S}_{2} \mathrm{O}_{8} 140$ $\mathrm{mM}$, ambos diluidos con agua ultrapura. Esta mezcla se almacenó durante 12-16 h en la oscuridad y luego se diluyó con una solución de metanol al 80\% hasta una absorbancia de 0,70 $\pm 0,02$ a $734 \mathrm{~nm}$. Después de agregar $10 \mu \mathrm{L}$ de Trolox (ácido 6hidroxi - 2, 5, 7, 8 - tetrametilcromo-2-ácido carboxílico [0 - $2000 \mu \mathrm{mol} / \mathrm{L}]$ o la muestra diluida a 2,5 $\mathrm{mL}$ de solución ABTS•+ diluida, se tomaron lecturas de absorbancia después de 7 min de la mezcla inicial con un espectrofotómetro UV-vis Cary-50.

\begin{tabular}{|l|c|}
\hline \multicolumn{1}{|c|}{ Extracto } & Capacidad Antioxidante ABTS•+ (Abs.: 734nm) \\
\hline A $(100 \%$ etanol $)$ & $102 \mu \mathrm{mol} \mathrm{TEAC*/g}$ \\
\hline B $(50 \%$ etanol $-50 \%$ agua destilada $)$ & $193 \mu \mathrm{mol} \mathrm{TEAC*/g}$ \\
\hline
\end{tabular}

Tabla 2. Capacidad Antioxidante de cada extracto.

*TEAC: capacidad antioxidante equivalente del Trolox. 
Teniendo en cuenta los resultados obtenidos en lo que respecta a la caracterización de los extractos en referencia a mayor contenido de polifenoles totales y mayor capacidad antioxidante se decidió continuar con el extracto etanólico-acuoso (Extracto B) para las siguientes etapas de la investigación.

Técnicas electroquímicas para la evaluación de la capacidad de inhibición de los extractos

Para estudiar la eficiencia del extracto etanólico-acuoso en la protección de los sustratos, se ensayaron probetas de acero SAE 1010 mediante técnicas electroquímicas (Aksut, Lorenz \& Mansfeld, 1982).

Curvas de polarización lineal.

Este ensayo se llevó a cabo sobre probetas de acero SAE 1010 a las cuales se les delimitó un área de $0,8 \mathrm{~cm}^{2}$. Se emplearon un contra electrodo de platino y un electrodo de calomel saturado (ECS) como electrodo de referencia; el electrolito soporte fue una solución de $\mathrm{NaCl}$ 0,5M.

Se realizó el ensayo de resistencia a la polarización del sustrato acero SAE 1010 con y sin orujo para los respectivos ensayos duplicados.

El rango de barrido fue de $\pm 20 \mathrm{mV}$ a partir del potencial de corrosión y se utilizó una velocidad de $1 \mathrm{mV} . \mathrm{s}^{-1}$. A partir de estos datos obtenidos se graficaron las curvas de resistencia a la polarización con/ sin compensación óhmica (Kelly, Scully, Shoesmith \& Buchheit, 2002).

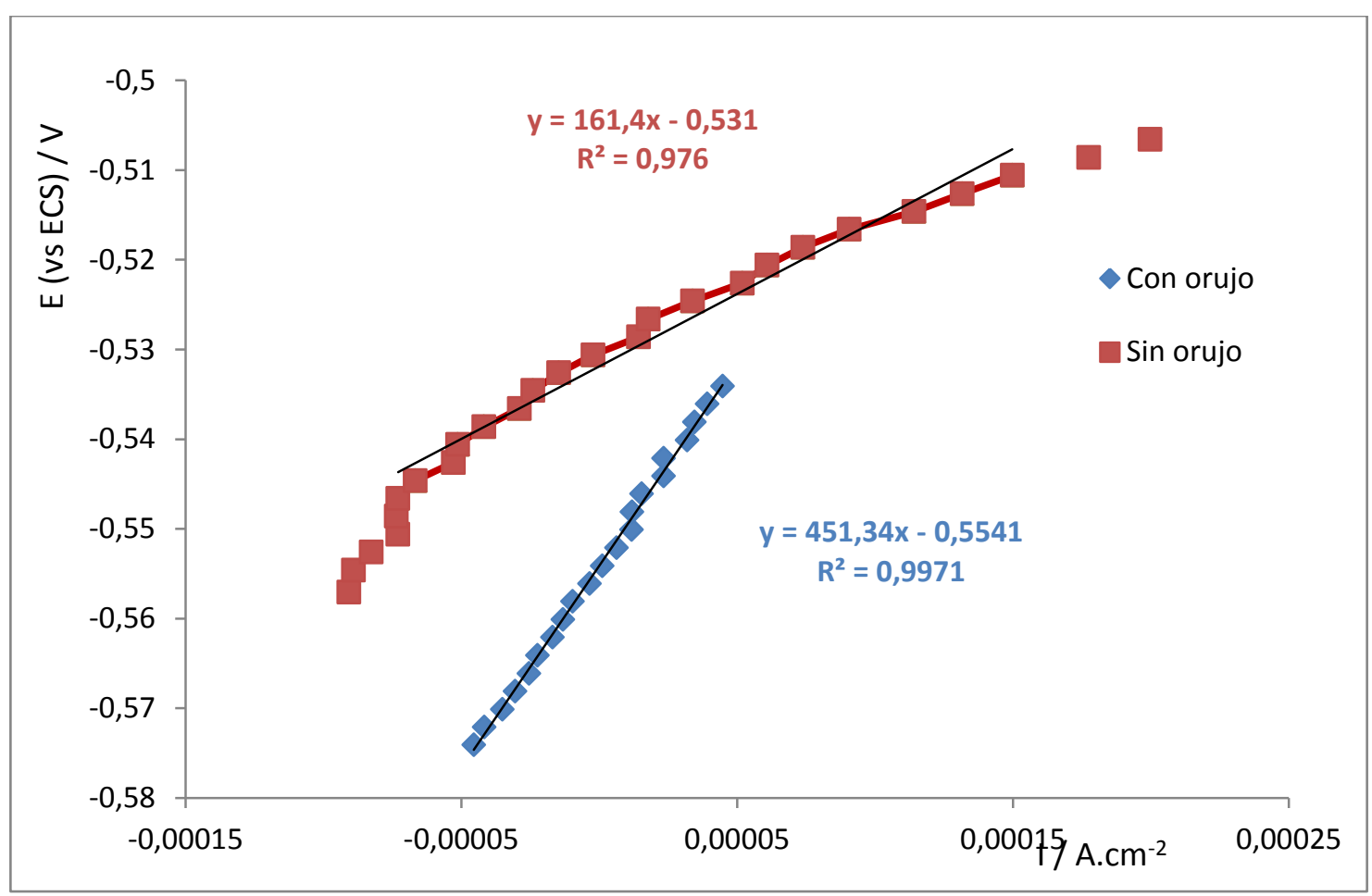

Figura 1: Curvas de polarización lineal: a) sin compensación por caída óhmica. 


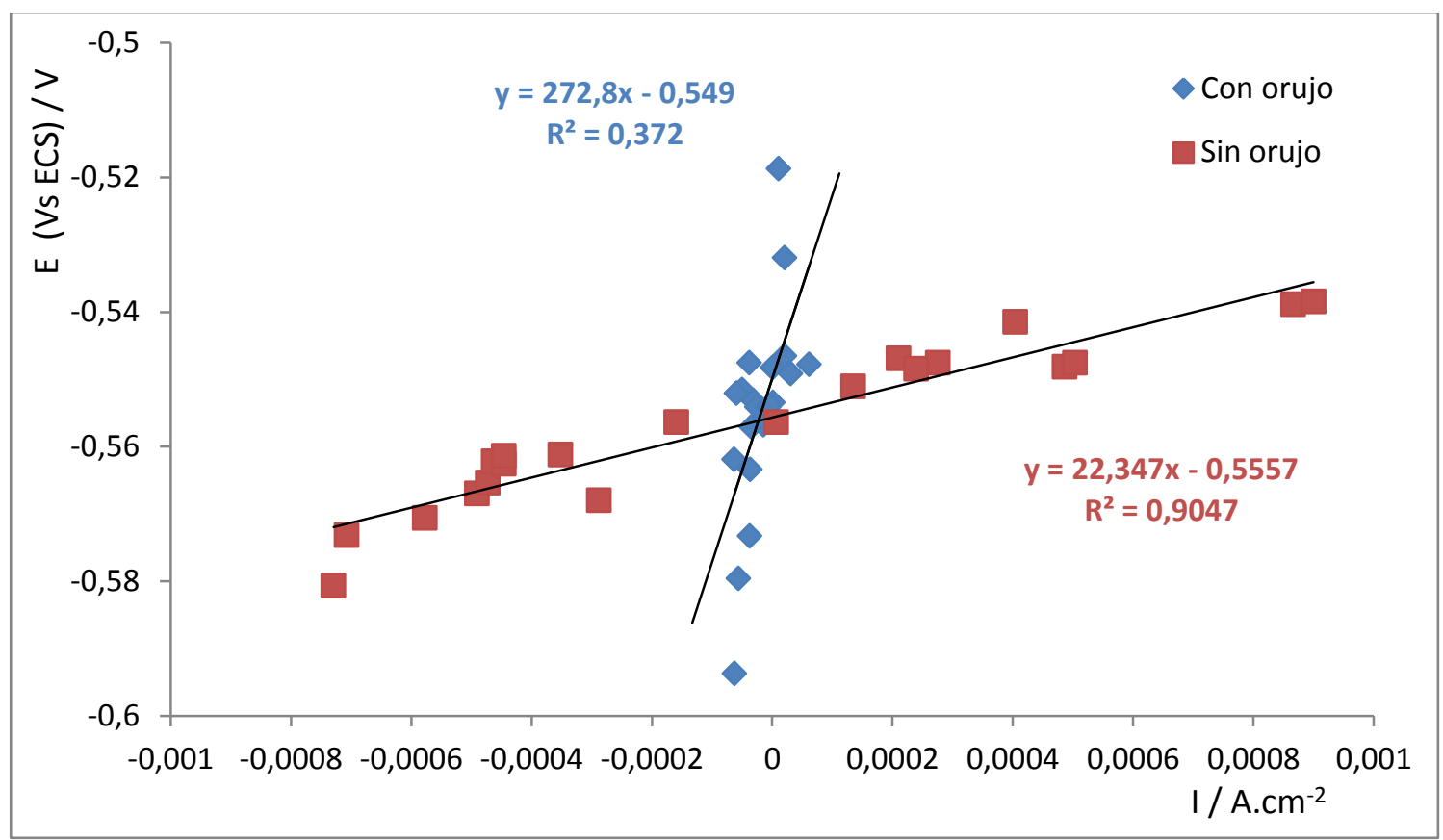

Figura 2: Curvas de polarización lineal: b) con compensación por caída óhmica.

Potencial de corrosión (Ecorr)

Para llevar a cabo la medición del potencial de corrosión se utilizaron como electrodos de trabajo probetas de acero SAE 1010 con un área de $0,8 \mathrm{~cm}^{2}$, en las mismas celdas anteriores. El potencial se midió, con agitación constante contra ECS. Las medidas se realizaron en forma continua durante 4 h y luego de 24 h de inmersión.

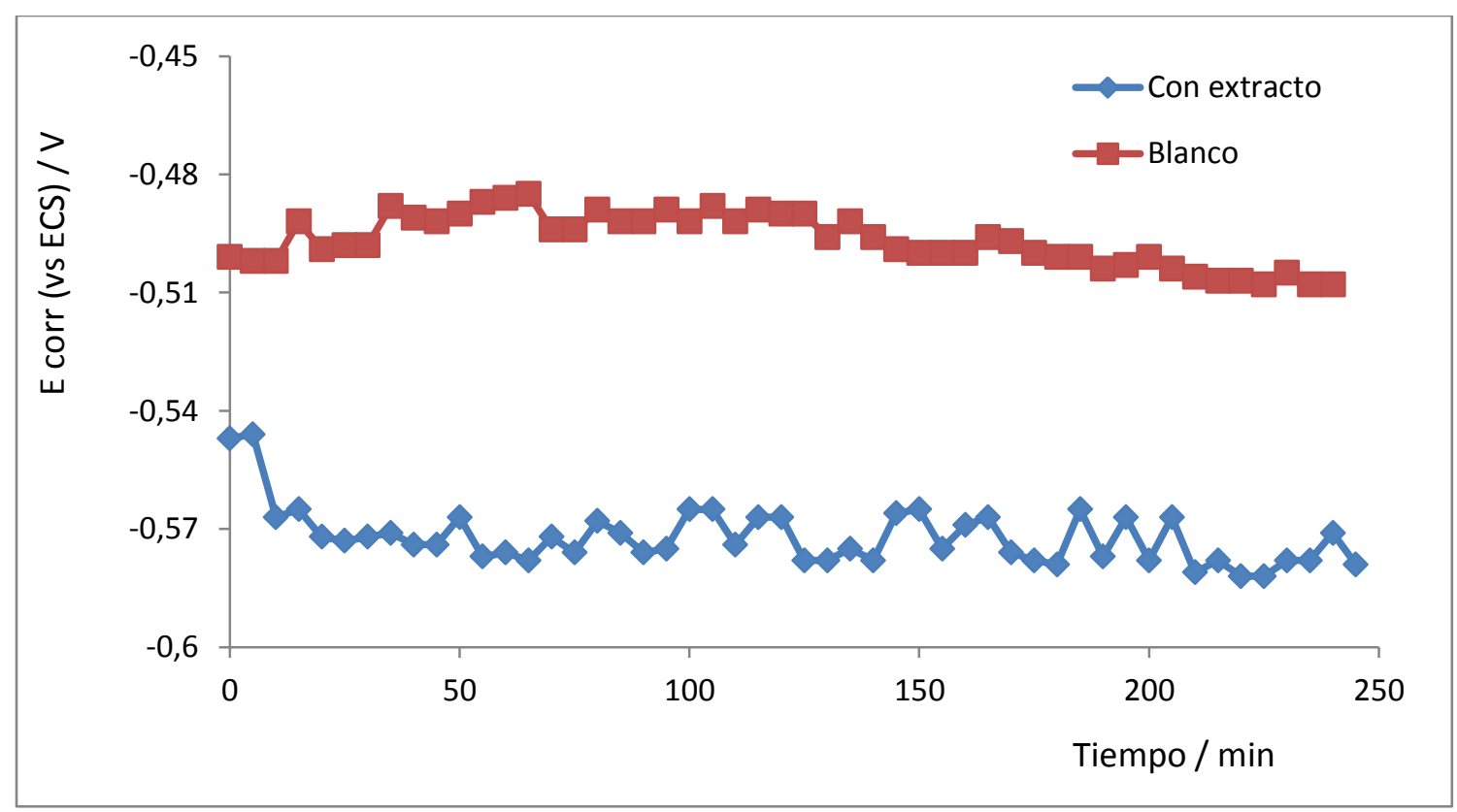

Figura 3: Potencial de Corrosión del acero SAE1010 con orujo y blanco en medio CINa. 


\section{Conclusiones}

Teniendo en cuenta los resultados obtenidos en lo que respecta a caracterización de los extractos, se decidió continuar con el extracto alcohólico - acuoso.

Las determinaciones de resistencia a la polarización muestran que la presencia del extracto etanol / agua, inhibe la corrosión del acero.

Las medidas de potencial de corrosión muestran que el acero se encuentra más activo cuando está en contacto con el orujo, probablemente debido a que se está llevando a cabo la formación de una película pasiva.

\section{Referencias}

Aksut, A., Lorenz, W. \& Mansfeld, F. (1982). Determination of corrosion rates by electrochemical d.c. and a.c. methods - II. Systems with discontinuous steady state polarization behavior. Corrosion Science 22, 611-619.

Alonso, J. (2000). The grape, webs.uolsinectis.com.ar/ fitomedicina/ RevMonografiaSolo.txt.html.

Antoniolli, A.; Fontana, A. R.; Piccoli, P. \& Bottini, R. (2015). Characterization of polyphenols and evaluation of antioxidant capacity in grape pomace of the cv. Malbec, Food Chemistry. 178: 172-178.

Bittner, A. (1989). Journal of Coatings Technology, 61 (777), 14-118.

Ciudad, C. \& Valenzuela, J. (2002). Antioxidantes en uvas viníferas y vino. Tierra adentro 42: 22-23.

Ferreyra, S.; Antoniolli, A.; Bottini, R. \& Fontana, A. (2019). Bioactive compounds and total antioxidant capacity of cane residues from different grape varieties. J Sci Food Agric. doi: 10.1002/ jsfa.10065.

Gerhard, A. \& Bittner, A. (1986). Journal of Coatings Technology, 58 (740), 59-65.

Kelly,R.; Scully, J.; Shoesmith, D. \& Buchheit, R. (2002). Electrochemical Techniques in Corrosion Science and Engineering, Marcel Dekker, Inc. ISBN: 0-8247-9917-8.

Kesavan, D. ; Gopiraman, M. \& Sulochana, N. (2012). Che Sci Rev Lett, 1(1), 1-8.

Romagnoli, R. \& Vetere, V. (1995). Corrosion (NACE), 51(2), 116-123.

Shirmohammadlia, Y., Efhamisisia, D. \& Pizzi, A. (2018).Tannins as a sustaintable raw material for green chemistry: A review, Industrial Crops \& Products 126, 316-332. 